\title{
Extension of the PSE code NOLOT for transition analysis in rotating reference frames
}

\author{
Xavier Dechamps and Stefan Hein
}

\begin{abstract}
In this paper, spatial linear stability analyses are performed on three flow configurations where rotational effects are present. The first two configurations are the two-dimensional flows along a flat plate and along a curved plate, both with a rotation vector along the spanwise direction. The third configuration is the threedimensional flow over a rotating disk with and without axial inflow. These flows are used as a verification of the extension of the stability analysis code NOLOT to rotating frames. For all these three flows, a perfect matching is observed in comparison with results from the literature for the neutral curves and the eigenfunctions. The present results show that rotation has a considerable effect on the stability of boundary layers. Depending on the intensity and orientation of the rotation vector, stabilization or destabilization of the boundary layer is observed.
\end{abstract}

\section{Introduction}

Flows over rotating geometries are encountered in a great number of aeronautical applications (e.g. propellers, rotors, wind turbines, helicopter blades, etc.). In most of these applications, the configuration exhibits rotating blades of some sort. In this case it is well-known that the laminar-turbulent transition is postponed in comparison with a non-rotating configuration (see [8] for a propeller configuration and [2,3] for more recent results on wind turbines). A physical interpretation is that the radial velocity component generated by the centrifugal force leads to an azimuthal Coriolis force which acts as a favorable pressure gradient and thus stabilizes the boundary

X. Dechamps · S. Hein

German Aerospace Center (DLR), Institute of Aerodynamics and Flow Technology, Bunsenstr. 10 37073 Göttingen, Germany

e-mail: Xavier.Dechamps@dlr.de

S. Hein

e-mail: Stefan.Hein@dlr.de 
layer. Moreover, the centrifugal force tends to make the boundary layer thinner in comparison with non-rotating cases. The main consequence is a delayed separation on the suction side.

The effects of rotation on the stability of boundary layers are only partially understood. One of the main open questions is concerned with the nature of the laminarturbulent transition. Does rotation add new kinds of instabilities or does it only alter transition mechanisms already existing in non-rotating cases? The present work aims at contributing to a better understanding of the effect of rotation on the stability properties of boundary layers. The instability of three generic and academic boundary layers exposed to to rotation is studied here. Out of the three flow configurations, the case of the rotating disk is the most interesting one. This flow is commonly studied because of its convenience to provide crossflow-dominated instabilities such as encountered on swept wings. In comparison with swept wings, the rotating disk is set up more easily experimentally since no wind tunnel is required and no leading edge contamination occurs as on a swept wing.

\section{Numerical method}

Very few instability analysis programs are conceived by considering compressibility, curvature, the growth of the boundary layer and rotation. NOLOT (NOnLOcal Transition analysis) is the result of a joint cooperation between DLR and the Swedish Defence Research Agency FOI and is based on an orthogonal curvilinear coordinate system. The Parabolized Stability Equations (PSE) based code NOLOT has been recently extended to rotating reference frames. The effect of the rotation vector $\boldsymbol{\Omega}$ is taken into account through the correction terms which are the Coriolis $2 \rho \boldsymbol{\Omega} \times \boldsymbol{U}$ and centrifugal $\rho \boldsymbol{\Omega} \times(\boldsymbol{\Omega} \times \boldsymbol{x})$ forces $(\boldsymbol{x}$ is the vector between the rotation vector and the considered point). The governing equations are made dimensionless by assuming a reference length $l_{0}^{*}$, a reference velocity $U_{0}^{*}$, a reference pressure $\rho_{0}^{*} U_{0}^{* 2}$ and a reference temperature $T_{0}^{*}$ (in the following all the starred quantities are dimensional and the subindex 0 denotes a reference value). During this nondimensionalization, dimensionless numbers appear naturally such as the Reynolds number $\operatorname{Re}=\left(U_{0}^{*} l_{0}^{*}\right) / v_{0}^{*}$, the Mach number Ma $=U_{0}^{*} / \sqrt{\gamma \mathscr{R} T_{0}^{*}}$, the Prandtl number $\operatorname{Pr}=\left(\mu_{0}^{*} c_{p 0}^{*}\right) / \kappa_{0}^{*}$ and the Rossby number Ro $=U_{0}^{*} /\left(2 \Omega_{0}^{*} l_{0}^{*}\right)$. The Rossby number represents the ratio between the inertial and Coriolis forces. The rotation number $\mathrm{N}=1 /(2 \mathrm{Ro})$ will also be used in the next section instead of the Rossby number.

The reader is referred to the work of Hein et al. [7] for the detailed implementation of the stability equations for boundary layers in NOLOT. Steady or unsteady perturbations propagate in a direction contained between the streamwise $x_{1}$ and spanwise $x_{2}$ directions. The normal-mode solution is assumed for the disturbance quantities and the amplitude functions have a slow variation along the streamwise direction. The perturbations on the velocity field $(\tilde{u}, \tilde{v}, \tilde{w})$, the temperature $\tilde{T}$ and the density $\tilde{\rho}$ take the following form for $\tilde{\mathbf{q}}=(\tilde{u}, \tilde{v}, \tilde{w}, \tilde{T}, \tilde{\rho})$ : 


$$
\tilde{\mathbf{q}}\left(x_{1}, x_{2}, x_{3}, t\right)=\hat{\mathbf{q}}\left(x_{1}, x_{3}\right) \exp \left(i\left(\int \alpha\left(x_{1}\right) \mathrm{d} x_{1}+\beta x_{2}-\omega t\right)\right)
$$

Herein $\omega$ is the real-valued angular frequency and $\alpha=\alpha_{r}+i \alpha_{i}$ is a complex number. The streamwise and spanwise components of the wavevector $\mathbf{k}$ are the real part of $\alpha$ $\left(=\alpha_{r}\right)$ and $\beta=\beta_{r}$. The angle between the wavevector $\mathbf{k}$ and the $x_{1}$-direction is equal to $\varepsilon=\tan ^{-1}\left(\beta / \alpha_{r}\right)$. The quantity $\sigma=-\alpha_{i}$ is identified as the spatial amplification rate along the $x_{1}$-direction. Four subsets of equations are implemented for local or non-local and for parallel or non-parallel computations. Local or non-local refers to whether the streamwise derivatives of the amplitude functions are kept or not in the stability equations. Parallel or non-parallel refers to whether the terms related to the non-parallel basic flow are kept or not. In the next section, only local computations are considered for the verification of the extension to a rotating frame.

\section{Numerical results}

In this section the stability analysis code including the rotational effect is executed on three kinds of boundary layers with increasing complexity. The first configuration considers the two-dimensional flow over a flat plate subjected to a spanwise-oriented rotation vector. The second configuration considers the same velocity field but this time along a curved wall subjected to a spanwise-oriented rotation vector placed at the center of curvature. The third configuration is the three-dimensional boundary layer over a rotating disk with and without axial inflow. For all these boundary layers, the corresponding compressible boundary layer equations are solved but the Mach number is chosen smaller than 0.1 . The purpose is to keep the flow essentially incompressible because only very few test cases combine rotation with compressibility in the literature, whereas incompressible rotating flows have been much more studied theoretically, numerically and experimentally.

\subsection{Flow along a flat plate with rotation}

This first configuration considers the two-dimensional boundary layer over a flat plate. The Blasius profile is used for the streamwise and wall-normal velocity components. These velocity components are subjected to a rotation vector aligned with the spanwise direction $x_{2}$ and centered at the footpoint of the considered boundary layer profile (see Fig. 1 of Potter and Chawla [11]). This configuration is artificial but aims at analysing fundamental effects of rotation. As stated by Potter and Chawla [11], the Coriolis term in the streamwise-momentum equation is negligible so that the Blasius equation can be used even with rotation. However the non-negligible contributions from rotation are kept in the stability equations. Local parallel stability analyses are performed at a fixed Reynolds number $\operatorname{Re}=350$ 
based on the velocity at the edge of the boundary layer $u_{\infty}^{*}$ and on the Blasius length $\delta^{*}=\sqrt{v^{*} x_{1}^{*} / u_{\infty}^{*}}$. These values were chosen in order to match the ones in Yecko and Rossi [14] where the reference length is the displacement thickness $\delta_{d}^{*}=1.7208 \delta^{*}$.

The left part of Fig. 1 shows the neutral curves for three values of the Rossby number in the plane $\left(\beta, \alpha_{r}\right)$. Ro $=-516,-1032$ and -3097 correspond to Ro $=$ 300,600 and 1800 in [14], the change in sign coming from the choice of another system of reference. The neutral curves from [14] are also shown for comparison. The growth rate is positive (unstable flow) inside the neutral curves and negative (stable flow) outside of them. The Tollmien-Schlichting (TS) instability, located at high $\alpha_{r}$ and small $\beta$, is seen to be only marginally affected by rotation. However the introduction of rotation leads to the appearance of a new region of instability at smaller $\alpha_{r}$ and higher $\beta$. This new region is much more dependent on the Rossby number than the TS instability. The size of the region corresponding to the rotational instability increases with the intensity of the rotation. The corresponding location of maximum growth rate is pushed to higher $\beta$ when the effect of rotation is increased.

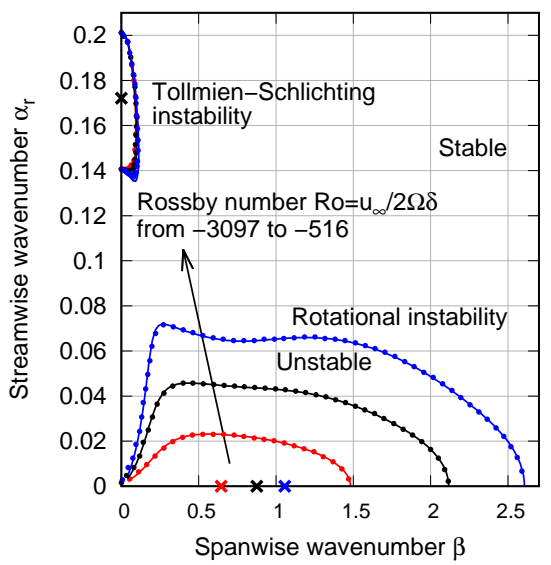

(a)

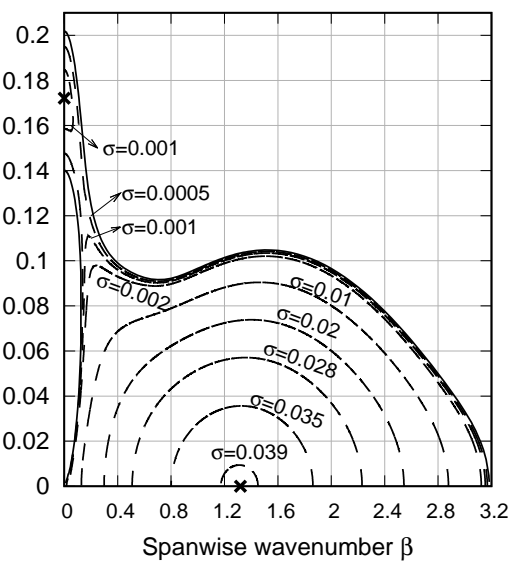

(b)

Fig. 1: Flow along a flat plate. (a) Neutral curves for 3 values of the Rossby number $($ Ro $=-516$, -1032 and -3097 correspond to Ro $=300,600$ and 1800 in [14]) in the plane $\left(\beta, \alpha_{r}\right)$ at a Reynolds number $\mathrm{Re}=350$. (full lines) Present numerical results and (dots) those of [14]. (b) Neutral curve (full line) and isovalues of the spatial growth rate $\sigma=-\alpha_{i}$ (dashed lines) for Ro $=-258$ in the same plane as (a). The crosses $\times$ denote the location of maximum growth rate for each instability.

The right part of Fig. 1 shows the neutral curve and isovalues of the spatial growth rate $\sigma=-\alpha_{i}$ for Ro $=-258$. At this intensity of rotation the regions of TS and rotational instabilities merge. The maximum growth rate for the rotational instability appears on the line $\alpha_{r}=0$ and is much higher than the maximum growth rate for the TS instability on $\beta=0$. This observation is valid for any Reynolds number. Thus 
in the case of the flow along a flat plate, rotation adds a new kind of instability that will predominate over the standard TS instability.

\subsection{Görtler instability with system rotation}

The flow along a curved plate (with a radius of curvature $R^{*}$ ) without rotation is subjected to the Görtler instability which appears as pairs of steady longitudinally elongated counter-rotating vortices (see the left part of Fig. 2). The distance between each pair of vortices is the wavelength $\lambda^{*}=2 \pi / \beta^{*}$. The instability motion of Görtler vortices is driven by centrifugal forces in association with the concave curvature. The same velocity field as in the previous section is used. The streamwise wavenumber and the temporal frequency are set to $\alpha_{r}=\omega=0$. The rotation vector is oriented along the spanwise direction $x_{2}$ and is located at the center of curvature. The Görtler instability is very sensitive to the type and location of the initial perturbations. The generation of neutral curves is thus troublesome because the local theory produces unphysical results at low Reynolds numbers and non-local computations provide different neutral curves for different initial perturbations (see e.g. [5]). For the sake of verifying the extension it was decided to focus on local parallel and non-parallel computations. The Görtler number defined in Eq. 2 is usually used to represent the neutral curves because it takes into account the Reynolds number and the curvature effect and physically represents the ratio between the centrifugal force due to the curvature and the viscous force. The Görtler vortices appear when the Görtler number exceeds a critical value. See Kim et al. [10] for a summary of values encountered in the literature.

$$
\mathrm{Go}=\frac{u_{\infty}^{*} \delta^{*}}{v^{*}} \sqrt{\frac{\delta^{*}}{R^{*}}}=\operatorname{Re} \sqrt{\frac{\delta^{*}}{R^{*}}}
$$

The right part of Fig. 2 shows the neutral curves of the Görtler instability for different values of the rotation number $N=\Omega^{*} \delta^{*} / u_{\infty}^{*}=-0.5,0,0.5$ and 2 . Numerical results from $[4,15]$ (local non-parallel) and [5] (non-local non-parallel) are also shown for comparison. In the short-wave region $\beta>1$, a perfect matching is observed between all the curves at identical rotation number. When the rotation vector is oriented opposite to the shear vorticity $(N<0)$, a stabilizing effect is observed because the neutral point is pushed towards higher Görtler numbers. On the contrary, a positive rotation number destabilizes the flow. In the long-wave region $\beta<1$ it is known that the local stability theory provides unphysical results for Görtler vortices and this explains the disparity in Fig. 2. In our case local parallel computations provide increasing Görtler numbers of the neutral points $\left(\mathrm{Go}_{n}\right)$ as $\beta$ decreases below 0.1 . In opposite local non-parallel computations provide monotonically decreasing Görtler numbers when $\beta$ decreases. One should also note a perfect matching between the present local non-parallel curve at $N=0$ and the one from [4] based on Smith's model [12] for all the values taken by $\beta$. At identical intensity of rotation $(\operatorname{abs}(N)=0.5)$, the increase in $\mathrm{Go}_{n}$ for a negative rotation number is bigger than 


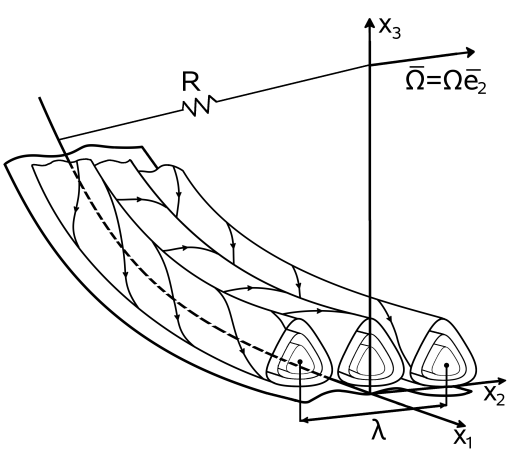

(a)

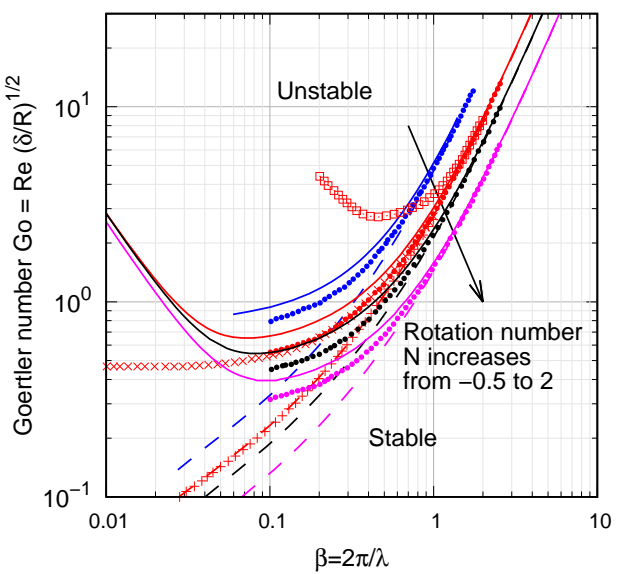

(b)

Fig. 2: Görtler instability with system rotation. (a) Geometry and (b) neutral curves in the plane $(\beta, \mathrm{Go})$ for four values of the rotation number $(N=-0.5,0,0.5$ and 2$)$. Present results based on $(-)$ local parallel theory, $(--)$ local non-parallel theory, $(\bullet)$ local non-parallel results from [15], $(+)$ local non-parallel result from [4] based on Smith's model [12] and $(\times)$ based on their own model and $(\cdot)$ one of the non-local non-parallel curves from [5].

the decrease in $\mathrm{Go}_{n}$ for a positive rotation number. This leads to the assumption that Görtler vortices are more easily stabilized than destabilized.

\subsection{Rotating disk with and without axial inflow}

The third flow configuration considers the three-dimensional incompressible boundary layer over a disk rotating at a constant rotation velocity $\Omega_{0}^{*}$ with and without axial inflow. This flow has been largely studied in literature because of its fundamental importance to model crossflow dominated transition. The rotating disk is characterized by a laminar region around the axis of rotation. At larger radial position a region of co-rotating vortices appears, immediately followed by a sharp transition to turbulence. The reference length $l_{0}^{*}$ is chosen equal to the radial position $x_{1}^{*}$ and the reference velocity is $U_{0}^{*}=\Omega_{0}^{*} x_{1}^{*}$ so that the Rossby number is $\mathrm{Ro}=0.5$ and the Reynolds number is $\operatorname{Re}=\left(\Omega_{0}^{*} x_{1}^{* 2}\right) / v_{0}^{*}$. When the disk is rotating inside an otherwise still fluid, the laminar-turbulent transition is known to occur at a local Reynolds number $\operatorname{Re}_{L}=\sqrt{\mathrm{Re}} \simeq 287$. The basic flow is calculated from the generalized von Kármán equations for a compressible flow (see e.g. [13]). These equations are solved in a transformed system of coordinates and an inverse Dorodnitsyn-Howarth transformation is applied to retrieve the compressibility information. If not explicitly stated otherwise, the stability analyses are local and non-parallel. 
The radial and azimuthal components of the basic flow are plotted in Fig. 3 as a function of the Dorodnitsyn-Howarth transformed wall-normal coordinate. The effect of an axial inflow is included through the parameter $T_{S}$ which corresponds to the ratio between the imposed axial inflow and the rotation speed of the disk. Without inflow $\left(T_{S}=0\right)$, an inflectional crossflow component is found. When the inflow contribution is increased, the crossflow component loses its inflectional nature and the thickness of the boundary layer decreases. From this first analysis one can postulate that an imposed axial inflow stabilizes the flow as it will be shown later.

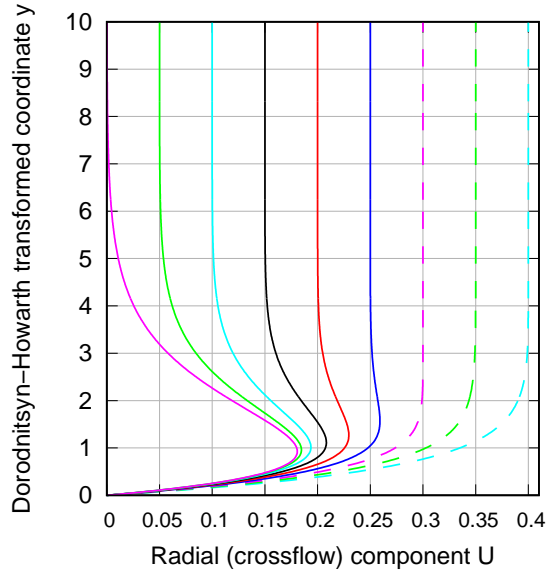

(a)

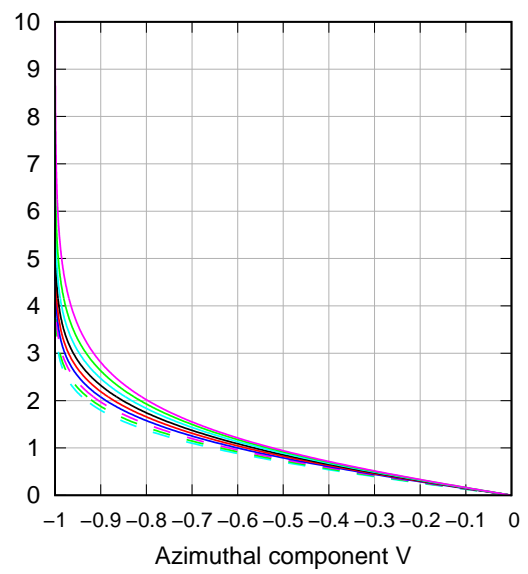

(b)

Fig. 3: Basic flow over a rotating disk as a function of the Dorodnitsyn-Howarth transformed wallnormal coordinate. (a) Radial (crossflow) component and (b) azimuthal component of the velocity field. The inflow parameter $T_{s}$ covers the range 0 to 0.4 by step of 0.05 from left to right for (a) and from right to left for (b).

Figure 4 shows the neutral curves for non-travelling perturbations $(\omega=0$ i.e. stationary relative to the rotating disk) and without axial inflow $\left(T_{S}=0\right)$. The $\mathrm{x}$-axis is the local Reynolds number and the $\mathrm{y}$-axes are the radial wave number $\alpha_{r}$ and wave angle $\varepsilon=\tan ^{-1}\left(\beta / \alpha_{r}\right)$. Numerical results from Hussain et al. [9] are also given for comparison as well as results obtained by neglecting the curvature effect in the stability equations and by cancelling the terms linked with rotation. When all the terms are kept in the equations, two types of instabilities appear. In the literature, the inviscid Type I instability is linked with the inflectional nature of the crossflow component of the basic flow and the viscous Type II instability is usually linked to the streamline curvature. These two instabilities have different critical Reynolds numbers. When the curvature and rotation effects are neglected, the Type II instability can not be predicted. As a matter of fact the Type II instability has a triple deck structure (see [6]) and its structure is fixed by a balance between viscous and Coriolis forces. Moreover, the critical Reynolds number is increased when the rotation 
terms are included. This might be linked with the radial components of the Coriolis and centrifugal forces which are both oriented towards the axis of rotation.

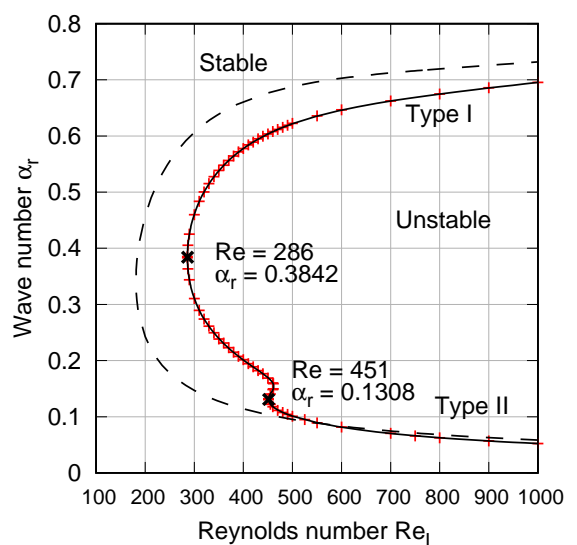

(a)

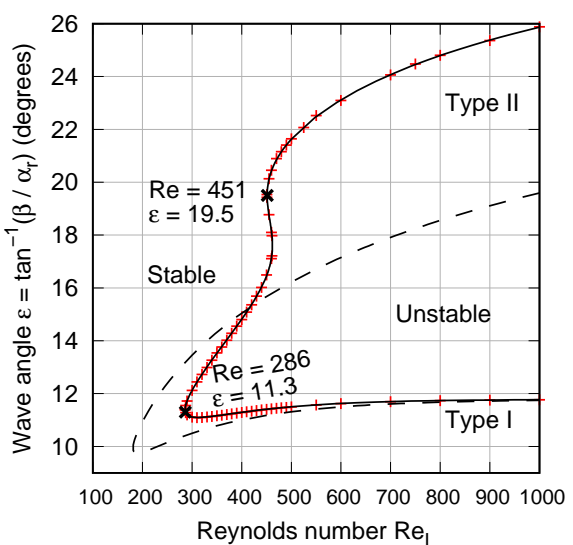

(b)

Fig. 4: Neutral curves for a rotating disk in the planes (a) $\left(\operatorname{Re}_{L}, \alpha_{r}\right)$ and (b) $\left(\operatorname{Re}_{L}, \varepsilon\right)$ for nontravelling disturbances without axial inflow. (-) Local non-parallel computations with curvature and rotation terms, $(--)$ local parallel computations without curvature and rotation terms, $(+)$ numerical results from [9].

The left part of Fig. 5 shows the neutral curves for travelling disturbances moving at nondimensional angular frequencies $\omega=2 \pi f^{*} / \Omega^{*}=-5,0,4$ and 7.9 relative to the disk without axial inflow. Numerical results from Hussain et al. [9] are also given for comparison. For positive frequencies the perturbations are propagating relative to the disk in the direction of the wave vector $\mathbf{k}$ and for negative frequencies opposite to it. In experiments non-travelling disturbances $(\omega=0)$ are nearly always observed because of their high sensitivity to surface roughness. However, travelling disturbances were also observed when highly polished and clean disks are used (see e.g. [1]). Major observations are that the Type II instability disappears for $\omega<0$ and high positive values of $\omega$ leads to a much lower critical Reynolds number for Type II. A variation of the frequency has a more restricted impact on the Type I instability. Another important point, which is not represented here, is that the growth rate $\sigma$ for $\omega<0$ is bigger than the one for $\omega>0$ at identical Reynolds number.

The right part of Fig. 5 shows the neutral curves of non-travelling disturbances within an axial inflow. The inflow parameter $T_{s}$ covers the values $0,0.05,0.15$, $0.25,0.3,0.35$ and 0.4 . The global effect of an axial inflow is to stabilize the flow as seen by the increase of the critical Reynolds number with $T_{s}$. Moreover, at identical Reynolds numbers, the growth rate also decreases when $T_{s}$ increases. The Type II instability becomes relatively important in comparison with the Type I instability. This is coherent with the facts that the crossflow component $U$ loses its inflectional 


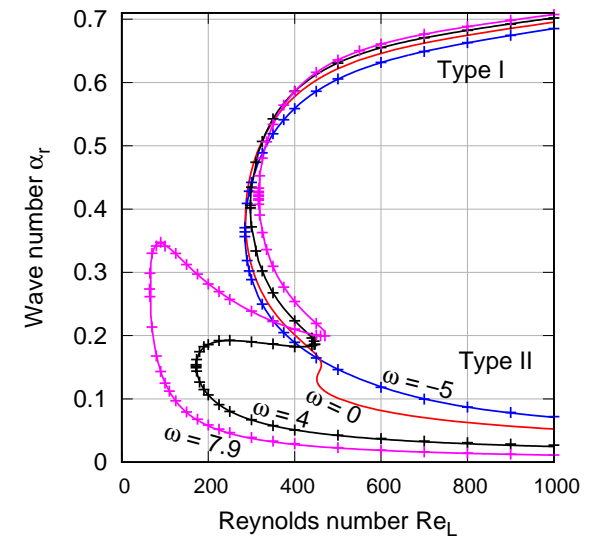

(a)

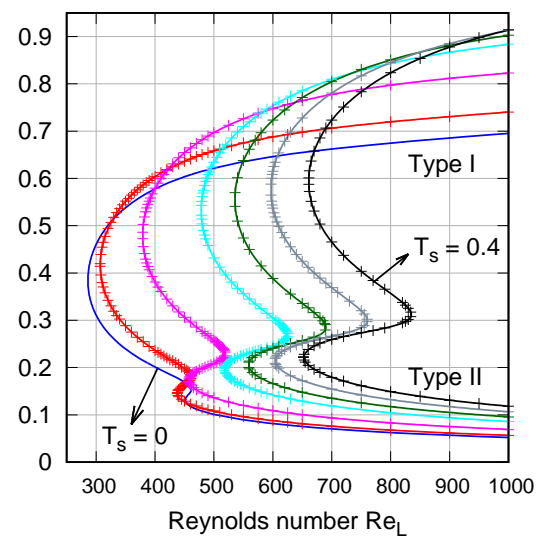

(b)

Fig. 5: Neutral curves for a rotating disk in the plane $\left(\operatorname{Re}_{L}, \alpha_{r}\right)$ for (a) travelling disturbances without axial inflow and (b) non-travelling disturbances with axial inflow $\left(T_{s}=0,0.05,0.15,0.25\right.$, $0.3,0.35$ and 0.4 from left to right). (+) Numerical results from [9].

nature and the thickness of the boundary layer decreases, as seen in Fig. 3. Thus a viscous mode of instability becomes predominant for sufficiently high values of $T_{s}$.

\section{Summary and outlook}

This paper focused on the study of three verification cases for the stability of boundary layers under the effect of rotation. In the case of the two-dimensional flow over a flat plate under the effect of rotation, it was observed that $\boldsymbol{\Omega} \cdot\left(\boldsymbol{\nabla} \times \boldsymbol{U}_{\text {basic }}\right)<0$ destabilizes the flow and generates a new kind of instability in addition to the standard Tollmien-Schlichting instability. For a sufficiently high rotation, the rotational instability with the highest growth rate has a wavevector perpendicular to the flow.

The stationary Görtler vortices under the effect of rotation showed on the contrary that $\boldsymbol{\Omega} \cdot\left(\boldsymbol{\nabla} \times \boldsymbol{U}_{\text {basic }}\right)>0$ destabilizes the flow. In this case the rotation does not add a new kind of instability but alters the characteristics of the already existing Görtler instability. Indeed a destabilizing rotation leads to higher growth rates, lower critical Reynolds numbers and smaller values for the most amplified wavelength $\Lambda=\operatorname{Go}(2 \pi / \beta)^{2 / 3}$.

The three-dimensional flow over a rotating disk puts forward two kinds of instabilities. In the case of non-travelling perturbations in an otherwise still fluid, the Type I crossflow instability has a lower critical Reynolds number and higher growth rate than the Type II instability. Perturbations with a negative temporal frequency do not show the Type II instability and have a higher growth rate than perturba- 
tions with positive temporal frequencies. In practice travelling disturbances are only dominating in experiments with highly polished and clean disks. An axial inflow stabilizes the boundary layer by increasing the critical Reynolds number of both types of instabilities and by decreasing their growth rates.

In the near future linear stability analyses will be applied to the boundary layer over a rotating propeller. From a geometrical point of view this configuration is very similar to the one of the rotating disk. For the practical application of the approach presented in this article it is fundamentally important to obtain answers for this type of an industrially relevant configuration. By switching on and off the contribution of rotation in the stability equations, one will observe the effect of rotation on the stability of this particular flow.

Acknowledgements The financial support of Airbus Operations GmbH is gratefully acknowledged. Moreover the authors would like to acknowledge the assistance of Alison Cooper (University of Warwick, UK) and Stephen J. Garrett (University of Leicester, UK) by providing detailed reference data for the rotating disk case.

\section{References}

1. Corke, T.C., Knasiak, K.F.: Stationary travelling cross-flow mode interactions on a rotating disk, J. Fluid Mech. 355, 285-315 (1998)

2. Du, Z., Selig, M.S.: The effect of rotation on the boundary layer of a wind turbine blade, Renewable Energy 20, 167-181 (2000)

3. Dumitrescu, H., Cardos, V.: Rotational effects on the boundary-layer flow in wind turbines, AIAA J. 42(2), 408-411 (2004)

4. Floryan, J.M., Saric, W.S.: Stability of Görtler vortices in boundary layers, AIAA J. 20(3), 316-324 (1982)

5. Hall, P.: The linear development of Görtler vortices in growing boundary layer, J. Fluid Mech. 130, 41-58 (1983)

6. Hall, P.: An asymptotic investigation of the stationary modes of instability of the boundary layer on a rotating disc, Proc. R. Soc. London, Ser. A 406, 93-106 (1986)

7. Hein, S., Bertolotti, F., Simen, M., Hanifi, A., Henningson, D.: Linear non-local instability analysis - The linear NOLOT code, DLR IB 223-94 A56, (1995)

8. Himmelskamp, H.: Profile investigations on a rotating airscrew, MAP Völkenrode, Reports and Translations 832 (1947)

9. Hussain, Z., Garrett, S.J., Stephen, S.O.: The instability of the boundary layer over a disk rotating in an enforced axial flow, Phys. Fluids 23(1), 114108 (2011)

10. Kim, M.C., Choi, C.K., Yoon, D.Y.: The onset of Görtler vortices in laminar boundary layer flow over a slightly concave wall, Eur. J. Mech. B-Fluid 29, 407-414 (2010)

11. Potter, M., Chawla, M.D.: Stability of boundary layer flow subject to rotation, Phys. Fluids 14(11), 2278-2281 (1971)

12. Smith, A.M.O.: On the growth of Taylor-Görtler vortices along highly concave walls, Q. Appl. Math. 13(3), 233-262 (1955)

13. Towers, P.D., Garrett, S.J.: Similarity solutions of compressible flow over a rotating cone with surface suction, Therm. Sci. 20(2), 517-528 (2016)

14. Yecko, P., Rossi, M.: Transient growth and instability in rotating boundary layers, Phys. Fluids 16(7), 2322-2335 (2004)

15. Zebib, A., Bottaro, A.: Goertler vortices with system rotation: linear theory, Phys. Fluids A 5(5), 1206-1210 (1993) 